\title{
Nomenclature and treatment of secondary urethral strictures following primary hypospadias repair: weighing up academic principles and clinical pragmatism
}

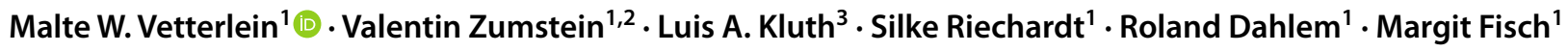

Received: 19 September 2020 / Accepted: 21 September 2020 / Published online: 6 October 2020

(c) The Author(s) 2020

\section{Dear Editor,}

With great interest we have read the comments by Drs. Shekar and Shivakumar [1] regarding our recent article on buccal mucosal graft urethroplasty (BMGU) for distal urethral strictures [2]. In that regard, we would like to put some points in perspective:

First, the authors have expressed some criticism about including patients who had previously undergone treatment for hypospadias. Hence, they claim that our report of a "homogeneous" cohort was untrue. While we concur with the notion that patients with hypospadias-associated strictures do embody a very specific subgroup with distinct disease characteristics, we would like to underline that the homogeneity of our series is warranted by the surgical technique (BMGU using a dorsal inlay) [2]. Contrary to the authors' opinion, we believe that the end does actually justify the means and that it is important to emphasize the feasibility and durability of one particular surgical technique in such population, especially given the abundance of techniques for the repair of the distal urethra. From a clinical

Malte W. Vetterlein and Luis A. Kluth: Member of the Trauma and Reconstructive Urology Working Party of the European Association of Urology (EAU) Young Academic Urologists (YAU).

This reply refers to the comment available online at https://doi. org/10.1007/s00345-020-03232-w.

Malte W. Vetterlein

malte.vetterlein@gmail.com

1 Department of Urology, University Medical Center Hamburg-Eppendorf, Hamburg, Germany

2 Department of Urology, Cantonal Medical Center St. Gallen, St. Gallen, Switzerland

3 Department of Urology, University Medical Center Frankfurt, Frankfurt/Main, Germany perspective, we are confident that it is reasonable to pragmatically approach and consider all patients for the decisionmaking process who present with urethral narrowing of the fossa navicularis or meatus, irrespective of the etiology. In our opinion, the nomenclature is of secondary importance here. Whether the urethral condition is labeled "obliteration", "narrowing", "stricture" or "stenosis" does not change the fact that the patient is in need of surgical intervention. Thus, the controversy regarding the correct nomenclature is more or less predominantly academic [3]. However, we absolutely agree that using internationally acknowledged staging systems might facilitate multi-institutional comparisons, first and foremost to evaluate the efficacy of different surgical techniques in discrete stricture cohorts. A great example is the recently developed LSE classification system based on stricture length (L), segment (S), and etiology (E) [4], which particularly accounts for a stricture in the segment of prior hypospadias repair and allows for organizing "[...] a heterogeneous condition [...] that will improve our ability to study the disease process" [4].

Second, as the editorialists correctly mention, we had initially refrained from stratifying stricture recurrence by etiology given the relatively small sample of 32 patients with only 10 recurrences and thus, limited room for interpretation. Nevertheless, we gladly provide those data here to complete the full picture and the results are depicted in Fig. 1. Overall, there was no statistical difference in recurrence rates between different etiologies $(P \geq 0.6)$. However, it should be noted that it is impossible to draw statistically meaningful conclusions from stratifying those ten patients according to etiology and larger study cohorts are needed to allow for valid inferences.

Finally, we entirely agree with the authors that eventually long-term outcomes should be reported to evaluate the durability of a surgical technique. Indeed, we have recently presented preliminary results from a cohort of 81 patients after primary hypospadias repair that underwent one-stage 


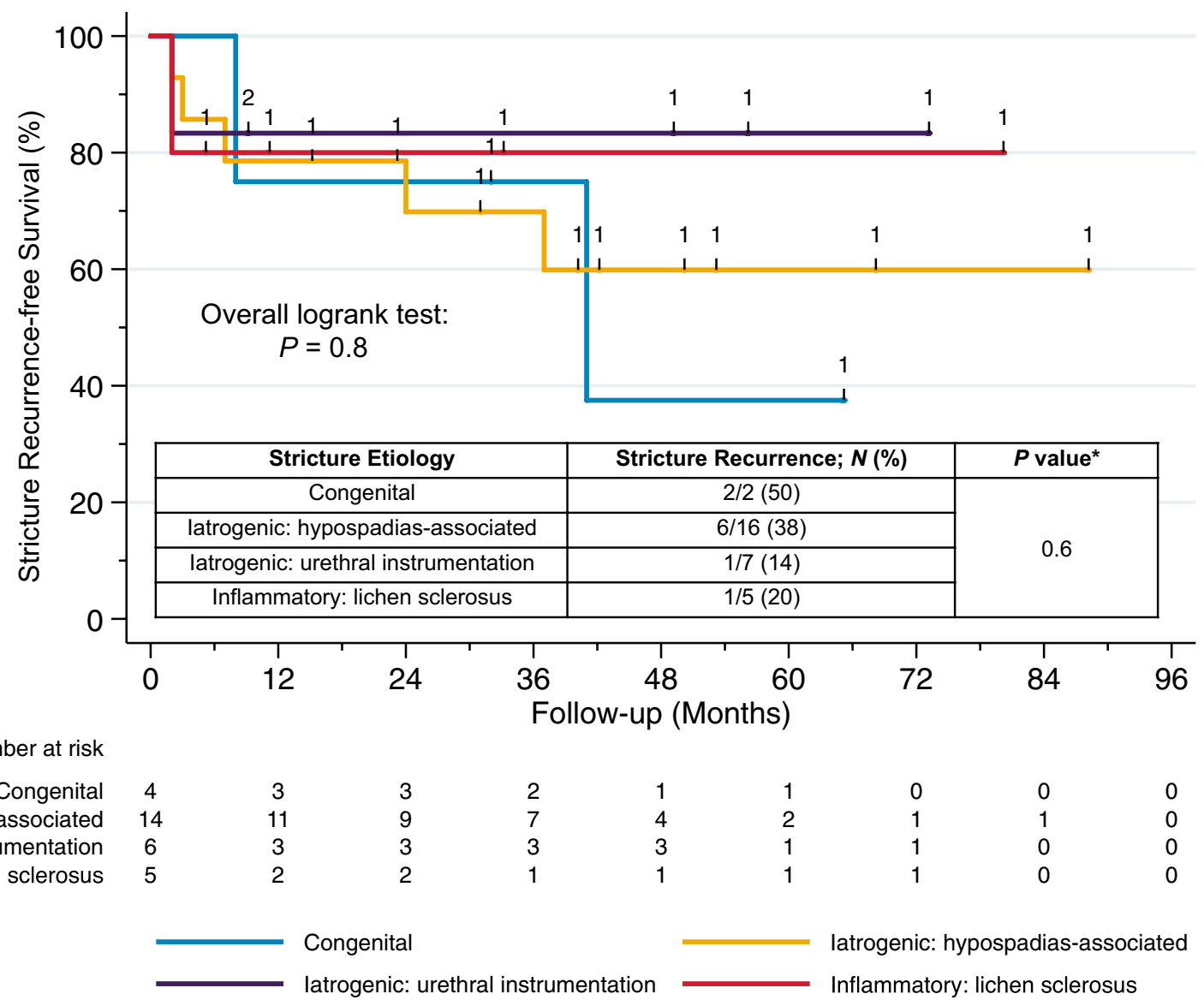

Fig. 1 Kaplan-Meier estimates of stricture recurrence-free survival in 32 men who underwent single-stage dorsal inlay BMGU stratified by stricture etiology ( $N=3$ were omitted due to short follow-up of $<2$ months). $B M G U$ buccal mucosal graft urethroplasty. *Fisher's exact test.

BMGU for secondary stricture. At a median follow-up of 70 months, 2-year and 5-year recurrence-free survival was $70 \%$ and $56 \%$, respectively, which translated into 5-year recurrence-free survival of $65 \%$ in the subgroup of patients presenting with meatal stenosis or fossa navicularis stricture [5]. Such data along with endeavors to collapse multi-institutional databases will help to better understand the distinct features and therapeutic requirements of patients with rare stricture locations, adverse etiology, and other complicating factors by increasing sample sizes and duration of follow-up.

Author contributions MWV: data collection and management, data analysis, manuscript writing. VZ: data collection, data analysis, manuscript writing. LAK: project development, data management, manuscript editing. SR: manuscript editing. RD: manuscript editing. MF: project development, manuscript editing.

Funding Open Access funding enabled and organized by Projekt DEAL.

\section{Compliance with ethical standards}

Conflict of interest All authors have no conflict of interest to disclose.

Statement of human rights All procedures performed in studies involving human participants were in accordance with the ethical standards of the institutional and national research committee and with the 1964 Helsinki Declaration and its later amendments or comparable ethical standards.

Informed consent Informed consent was obtained from all individual participants included in the study.

Open Access This article is licensed under a Creative Commons Attribution 4.0 International License, which permits use, sharing, adaptation, distribution and reproduction in any medium or format, as long as you give appropriate credit to the original author(s) and the source, provide a link to the Creative Commons licence, and indicate if changes were made. The images or other third party material in this article are included in the article's Creative Commons licence, unless indicated otherwise in a credit line to the material. If material is not included in the article's Creative Commons licence and your intended use is not permitted by statutory regulation or exceeds the permitted use, you will 
need to obtain permission directly from the copyright holder. To view a copy of this licence, visit http://creativecommons.org/licenses/by/4.0/.

\section{References}

1. Shekar PA, Shivakumar KS (2020) Can "strictures" following hypospadias repair be called true strictures?: the need for redefining terminology. World J Urol. https://doi.org/10.1007/s0034 5-020-03232-w

2. Zumstein V, Dahlem R, Maurer V et al (2020) Single-stage buccal mucosal graft urethroplasty for meatal stenoses and fossa navicularis strictures: a monocentric outcome analysis and literature review on alternative treatment options. World J Urol 38:26092620. https://doi.org/10.1007/s00345-019-03035-8

3. Latini JM, McAninch JW, Brandes SB et al (2014) SIU/ICUD consultation on urethral strictures: epidemiology, etiology, anatomy, and nomenclature of urethral stenoses, strictures, and pelvic fracture urethral disruption injuries. Urology 83:S1

4. Erickson BA, Flynn KJ, Hahn AE et al (2020) Development and validation of a male anterior urethral stricture classification system. Urology 143:241

5. Vetterlein MW, Stahlberg J, Bahassan O et al (2020) The role of one-stage buccal mucosal graft urethroplasty for secondary anterior urethral strictures following failed primary hypospadias repair: Long-term outcomes from a high volume referral center. Eur Urol Open Sci 19:e364

Publisher's Note Springer Nature remains neutral with regard to jurisdictional claims in published maps and institutional affiliations. 